\title{
“Reencontrándome a través de la diálisis peritoneal": Un abordaje fenomenológico
}

\author{
Yenny Fernanda Jiménez ${ }^{1}$, Gloria Mabel Carrillo² \\ ${ }^{1}$ Enfermera. Magister en Enfermería. Especialista en enfermería, cuidado Nefrológico y Urológico, Clínica Me- \\ dilaser S.A \\ ${ }^{2}$ Phd. MsC. Profesora Asociada Universidad Nacional de Colombia. Sede Bogotá. Facultad de Enfermería. Colombia
}

\section{Resumen}

Objetivo: Describir la experiencia de personas con enfermedad renal crónica en terapia de diálisis peritoneal que asisten a una unidad renal de Huila-Colombia.

Material y Método: Investigación cualitativa fenomenológica descriptiva, se realizaron entrevistas en profundidad a 16 participantes que cumplieron los criterios de inclusión. El análisis se realizó a partir del abordaje de Collaizzi.

Resultados: 361 descripciones significativas, 94 unidades de significado, y 6 temas comunes: enfrentando la realidad, pérdidas, corporalidad, limitaciones, soporte y ganancias, describen la experiencia.

Conclusiones: Padecer una enfermedad renal crónica terminal representa la lucha que tiene el paciente frente a los cambios en su aspecto físico, el auto reconocimiento y las limitaciones que tiene el servicio de salud del país, sin embargo, se refleja también, la transformación tras aceptar la condición, involucrando la terapia dialítica a su vida, permitiendo reencontrarse a través de ella.

PALABRAS CLAVE: diálisis peritoneal; atención de enfermería; investigación cualitativa.

Correspondencia:

Yenny Fernanda Jiménez

Clínica Medilaser S.A

Avenida Los Muiscas. Tunja. Boyacá. Colombia

E-mail: fernandajimenezmoreno@yahoo.es

\section{"Reencountering me through peritoneal dialysis": A phenomenological approach}

\section{Abstract}

Objetivo: Objective: To describe the experience of people with chronic kidney disease in peritoneal dialysis therapy attending a renal unit in Huila-Colombia.

Materials and methods: Descriptive phenomenological qualitative research. In-depth interviews were conducted with 16 participants who met the inclusion criteria. The analysis was made based on the Collaizzi approach.

Results: 361 significant descriptions, 94 units of meaning, and 6 common themes: facing reality, losses, corporality, limitations, support and gains, describe the experience.

Conclusions: Having a terminal chronic kidney disease represents the patient's struggle against changes in physical appearance, self-recognition and the limitations of the country's health service. However, the transformation is also reflected after accepting the condition, involving dialysis therapy to the patient's life, allowing them to rediscover themselves.

KEYWORDS: peritoneal dialysis; nursing care; qualitative research.

"A pesar de todo, mi vida ha podido continuar, hay momentos tristes pero no todo es así, también me siento feliz porque de no ser por la diálisis yo no sé dónde estaría..., hoy estoy vivo con mi familia y tengo alientos de seguir adelante" $(P) 1,(E)$ IJFG 


\section{Introducción}

Vivir con una enfermedad crónica enfrenta al paciente al proceso de adaptación y cambio de rol, trae consigo cambios físicos manifestados a través de síntomas, afectando los ámbitos de la vida: físico, emocional, familiar, social y económico replanteando la percepción respecto a si mismo ${ }^{1}$.

La enfermedad renal crónica afecta cerca del $10 \%$ de la población del mundo entero, por lo cual se ha considerado como una amenaza mundial; en Colombia su prevalencia es de 621 pacientes por millón de habitantes. El Huila tiene una prevalencia de $86,9 \%$ de personas con ERC ocupando el cuarto puesto de las ciudades capitales con mayor cantidad de casos presentados, situación que es alarmante y que va en ascenso ${ }^{2}$.

Actualmente el departamento del Huila cuentan con 6 unidades renales, la ciudad de Neiva tiene cinco; estas brindan atención y ofrecen tratamiento renal sustitutivo, mediante hemodiálisis y diálisis peritoneal, a las personas con enfermedad renal crónica; también se dispone de consulta de Enfermedad Renal Cónica Avanzada (ERCA), para aquellas personas con afectación renal temprana (filtrado glomerular menor a 60 $\mathrm{ml} / \mathrm{min}$ ), cubriendo así tanto a los usuarios del régimen contributivo como los subsidiados. La unidad renal donde se llevó a cabo el estudio atiende a 108 personas en hemodiálisis, 43 en Diálisis Peritoneal y 22 en consulta ERCA, dato que es fluctuante debido a que la población va en aumento y presenta un alto índice de mortalidad ${ }^{3}$.

En el caso específico de la Enfermedad Renal Crónica (ERC), la pérdida de la función renal y la dependencia a la terapia renal sustitutiva, supone cambios importantes en la vida del enfermo, su cotidianidad deberá adaptarse a los tiempos que conlleva realizar los intercambios en el caso de la diálisis peritoneal continua ambulatoria (DPCA), o descansar en la noche conectado a una maquina encargada de la terapia dialítica, en la modalidad de diálisis peritoneal automatizada (DPA). En el caso de existir alguna limitación es posible que la realización de la técnica dependa de un cuidador, responsabilidad altamente demandante ${ }^{4}$.

La responsabilidad de cuidado para la familia, a medio y largo plazo, puede provocar situaciones de crisis, ya que exige una dedicación constante al enfermo $y$, en ocasiones, descuido de sus actividades propias ${ }^{5}$. En algunos casos se produce cambio de roles cuando el individuo enfermo es el principal soporte de la familia y debe ser remplazado por otro miembro, ocasionándole a éste frustración y sufrimiento6,7, reflejándose en una alteración de la dinámica familiar.

Para el personal de enfermería es indispensable la interacción con el paciente, identificando sus particularidades, actitudes, intereses, motivaciones, miedos, destrezas. Ello es posible cuando se tiene al individuo como emisor de la información y se ha construido un vínculo estrecho entre cada una de las partes, generando confianza, seguridad y apoyo mutuo ${ }^{8}$.

Conocer la experiencia de los pacientes con enfermedad renal crónica en diálisis peritoneal permite identificar el trasfondo de convivir con la terapia de remplazo renal, reconocer los aspectos significativos al enfrentarse a una terapia que requiere entrenamiento, manejo en el domicilio y poca supervisión diaria del equipo de salud con el objetivo de lograr una mejor atención de los mismos. Es importante explorar las dinámicas de vida de personas con enfermedades crónicas en sus diferentes áreas de expresión, y comprender cómo sus actores participan en sus procesos de salud, como vía para potenciar el bienestar, la salud y la calidad de vida?

El objetivo del estudio fue describir la experiencia de personas con enfermedad renal crónica en terapia de diálisis peritoneal que asisten a una unidad renal de Huila-Colombia.

\section{Material y Método}

Estudio de abordaje cualitativo, tipo fenomenológico descriptivo, permitiendo estudiar el fenómeno como parte del cuidado de la experiencia humana, tal y como estos se presentan en el individuo. Reconociendo el fenómeno viviente tal como el existe, dando así un valor prioritario, único a su mundo ${ }^{10}$.

La recolección de la información se realizó a través de entrevistas a profundidad con las siguientes preguntas orientadoras: 1) Describa cómo ha sido la experiencia de vivir con esta terapia renal de diálisis peritoneal, desde que usted recuerda que inicio esta. 2) Trate de recordar cómo ha sido su experiencia en cada momento de la situación.

Se concertaron dos encuentros con los participantes, se mantuvo la técnica de conversación entre iguales, logrando establecer un vínculo con cada uno de ellos, facilitando la recolección de la experiencia del fenómeno, a 
través de la realización de 16 entrevistas a profundidad. En el primer encuentro, se dio a conocer el fenómeno, se firmó el consentimiento informado, se registraron los datos socio-demográfico y demás información contemplada en el instrumento de caracterización del paciente con ECNT del Grupo de Cuidado al Paciente crónico de la Universidad Nacional de Colombia previo consentimiento para su aplicación, en el segundo encuentro se desarrolló la entrevista a profundidad con el participante, la cual fue gravada con previo consentimiento del participante.

Fueron criterios de inclusión: 1. Adulto que acepte participar en la investigación y que firme el consentimiento informado, 2. Diagnóstico de enfermedad renal crónica terminal, vinculados al programa de diálisis peritoneal. 3. Sin déficit cognitivo o enfermedad mental. 4. Residentes en Neiva (Colombia) y sus alrededores.

16 participantes se incluyeron en el estudio, acudiendo al criterio de saturación teórica mediante un muestreo teórico. Se realizaron 16 entrevistas a profundidad con una duración mínima de 50 minutos y máxima de 120 minutos, logrando un tiempo de grabación total de 1.520 minutos. Los datos se almacenaron en una "unidad hermenéutica" mediante el programa de ATLAS TI, versión 7 .

La validación de la información, se realizó con tres participantes a los que se dio a conocer los resultados que describen la experiencia.

Se contó con el consentimiento informado por los participantes, se tuvo aval por parte del comité de ética de la Universidad Nacional de Colombia, en sesión del 22 de mayo de 2017 y Acta 8 y de la institución de salud donde se captaron los participantes. Durante la investigación se protegieron los derechos y el bienestar de los sujetos de investigación, de acuerdo con la pauta 13 establecida por el Consejo de Organizaciones Internacionales de Ciencias Médicas CIOMS ${ }^{11}$.

Se mantuvieron los criterios de veracidad, reflexividad y verificación de la información ${ }^{12}$. (Tabla 1)

Tabla 1. Estrategias empleadas para garantizar el rigor metodológico en la investigación.

Criterios de Rigor Metodológico

Veracidad

Reflexibilidad:

Verificación de la Información:
Estrategias usadas en la investigación

- Se realizó las transcripciones textuales de las entrevistas una a una, inmediatamente estas fueron terminadas.

- Se analizó la información fielmente a medida que se recogía. Esto permitió la comparación permanente entre cada participante con él mismo.

- Se realizaron entrevistas a profundidad, con miras a consolidar su experiencia en diálisis peritoneal.

- Se permitió que los participantes expresaran sus sentimientos a través de la realización de entrevistas a profundidad.

- Selección de las participantes por la investigadora según los criterios de inclusión definidos sin ningún tipo de intermediarios.

- La selección progresiva de los participantes se hizo teniendo en cuenta los criterios de inclusión previamente definidos.

- El número de participantes estuvo determinado por la información recolectada con los actores del estudio (muestreo teórico), las entrevistas se realizaron hasta que se logró la saturación teórica.

- La información se recolectó y analizó en el período establecido por los investigadores.

- Se realizó la grabación y transcripción textual de todas las entrevistas que se desarroIlaron.

- Se describió el contexto del estudio y las características de los participantes.

- Se mantuvo la distancia prudencial entre las participantes y la investigadora con el fin de garantizar que éstas no manipulen o acomoden la información que brinden.

- Se describió el modo en que se relacionan las categorías que emergieron con la literatura.

- Se describió las características socio-demográficas: género, edad, estado civil, contexto familiar y social. Además, se recolectó información sobre el tiempo de ocurrencia de la enfermedad. De esta manera, los lectores o nuevos investigadores podrán analizar el contexto y determinar la adecuación de los hallazgos. 
Para la interpretación metodológica del estudio se seleccionó el abordaje de Colaizzi, quien refiere que para ampliar el conocimiento de un fenómeno, primero deben conocerse las fundamentaciones del mismo, estableciendo la definición del fenómeno de interés con base en la recolección de descripciones de los participantes, seguido del análisis secuencial para depurar la esencia de la información y validación, con los mismos participantes para llegar a elaborar una descripción exhaustiva del fenómeno a estudiar ${ }^{13}$. (Figura 1)

En la Tabla 2 podemos observar las características sociodemográficas de los 16 participantes en este estudio.

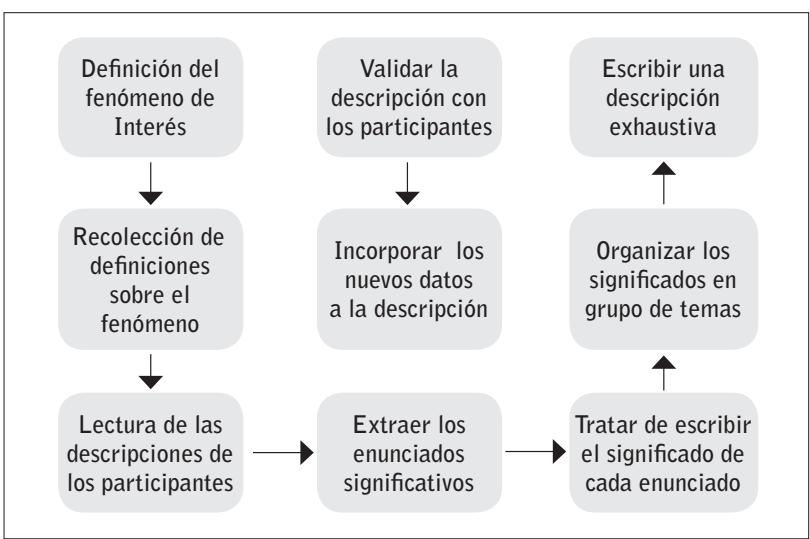

Proceso de análisis aplicado en la investigación, según Colaizzi. Fuente: Sánchez B. ${ }^{13}$

Tabla 2. Caracterización socio demográfica de los participantes.

\begin{tabular}{|c|c|c|c|c|c|c|c|c|c|}
\hline Participante & Género & Edad & Dx Médico & $\begin{array}{c}\text { Terapia } \\
\text { dialítica } \\
\text { Actual }\end{array}$ & Ocupación & $\begin{array}{l}\text { Seguridad } \\
\text { social }\end{array}$ & Estado civil & $\begin{array}{l}\text { Grado de } \\
\text { escolaridad }\end{array}$ & $\begin{array}{l}\text { Estrato } \\
\text { socio } \\
\text { económico }\end{array}$ \\
\hline 1 & $\mathrm{~F}$ & 61 & HTA, ERC & APD & Hogar & Subsidiado & Separada & Ninguno & 1 \\
\hline 2 & $M$ & 68 & $\begin{array}{l}\text { DM, HTA, } \\
\text { ERC }\end{array}$ & APD & Hogar & Subsidiado & Casado & $\begin{array}{l}\text { Primaria } \\
\text { incompleta }\end{array}$ & 2 \\
\hline 3 & $M$ & 65 & HTA, ERC & APD & Agricultor & Subsidiado & Casado & $\begin{array}{l}\text { Primaria } \\
\text { incompleta }\end{array}$ & 1 \\
\hline 4 & M & 57 & $\begin{array}{c}\text { DM, HTA, } \\
\text { ERC }\end{array}$ & APD & Hogar & Subsidiado & Unión libre & Ninguno & 1 \\
\hline 5 & $\mathrm{M}$ & 51 & HTA, ERC & APD & Agricultor & Subsidiado & Separada & $\begin{array}{c}\text { Secundaria } \\
\text { completa }\end{array}$ & 3 \\
\hline 6 & $\mathrm{~F}$ & 52 & HTA, ERC & APD & independiente & Contributivo & Viuda & Ninguno & 1 \\
\hline 7 & $\mathrm{~F}$ & 76 & $\begin{array}{l}\text { DM, HTA, } \\
\text { ERC }\end{array}$ & APD & Hogar & Subsidiado & Separada & $\begin{array}{l}\text { Secundaria } \\
\text { incompleta }\end{array}$ & 2 \\
\hline 8 & M & 68 & $\begin{array}{l}\text { DM, HTA, } \\
\text { ERC }\end{array}$ & APD & Hogar & Contributivo & Separada & $\begin{array}{l}\text { Secundaria } \\
\text { incompleta }\end{array}$ & 1 \\
\hline 9 & $\mathrm{~F}$ & 26 & $\begin{array}{l}\text { DM, HTA, } \\
\text { ERC }\end{array}$ & APD & Hogar & Subsidiado & Soltera & $\begin{array}{l}\text { Primaria } \\
\text { completa }\end{array}$ & 3 \\
\hline 10 & $\mathrm{~F}$ & 50 & $\begin{array}{l}\text { DM, HTA, } \\
\text { ERC }\end{array}$ & APD & Independiente & Subsidiado & Casada & Ninguno & 1 \\
\hline 11 & $\mathrm{~F}$ & 43 & HTA, ERC & APD & Independiente & Contributivo & Casada & $\begin{array}{l}\text { Secundaria } \\
\text { completa }\end{array}$ & 1 \\
\hline 12 & $\mathrm{~F}$ & 37 & HTA, ERC & APD & Hogar & Subsidiado & Separada & $\begin{array}{l}\text { Secundaria } \\
\text { completa }\end{array}$ & 1 \\
\hline 13 & $\mathrm{~F}$ & 65 & HTA, ERC & APD & Hogar & Subsidiado & Separada & Ninguno & 2 \\
\hline 14 & $\mathrm{~F}$ & 34 & $\begin{array}{l}\text { HTA, ERC, } \\
\text { LES }\end{array}$ & APD & Independiente & Subsidiado & Casada & $\begin{array}{l}\text { Secundaria } \\
\text { incompleta }\end{array}$ & 3 \\
\hline 15 & $\mathrm{~F}$ & 56 & HTA, ERC & APD & Independiente & Subsidiado & Separado & $\begin{array}{l}\text { Primaria } \\
\text { incompleta }\end{array}$ & 1 \\
\hline 16 & $\mathrm{~F}$ & 73 & HTA, ERC & APD & Hogar & Subsidiado & Casada & Ninguno & 1 \\
\hline
\end{tabular}

HTA: Hipertensión Arterial, DM: Diabetes Mellitus tipo II, LES: Lupus Eritematoso Sistémico, ERC: Enfermedad Renal Crónica. 


\section{Resultados}

La experiencia de las personas con enfermedad renal crónica en diálisis peritoneal representa la lucha constante que enfrenta el individuo enfermo y su familia, desde el momento del diagnóstico de la enfermedad, con miras a incorporar la terapia de remplazo renal en sus vidas $y$ adecuarlo a su cotidianidad. Refleja las limitaciones que tiene el sector salud para garantizar la idoneidad de su terapia dialítica, así como la importancia del soporte social familiar y del equipo de salud de la unidad renal sobre el afrontamiento y el éxito del tratamiento. Se describen los beneficios de la terapia de diálisis peritoneal, por ser ambulatoria, brindándole autonomía e independencia al enfermo, de esta manera él logra aceptar su nueva condición de salud, permitiéndole recuperar poco a poco su nuevo rol de vida.

Se identificaron 361 descripciones significativas, y 94 unidades de significado, dieron origen a 6 temas comunes: Enfrentando la realidad, pérdidas, corporalidad, limitaciones, soporte y ganancias.

Enfrentando la realidad es un tema que describe el impacto que tiene la enfermedad sobre el paciente y cómo influye en su vida, tanto física, como emocionalmente, aprender a sobrellevar la sintomatología y el proceso de aceptar la diálisis como tratamiento definitivo. Las unidades de significado síntomas de la enfermedad, la diálisis como única opción, conductas de riesgo previas y la enfermedad como castigo, dan cuenta de los sentimientos ambivalentes y de cuestionamientos que envuelve la vida de estas personas, al experimentar la sintomatología propia de la enfermedad y reconocer la importancia de iniciar la terapia de remplazo renal, sin embargo estos individuos al inicio del proceso, no logran entender las implicaciones que esto conlleva y deben replantear la percepción de muchas cosas en sus vidas.

"Cuando supe que me tocaba hacerme diálisis, fue como si el mundo se me acabara, como si se derrumbara, porque de un momento para el otro mi vida cambio" $(P)$ 13, (E) 13 YMS.

"Empezaron a hincharse los pies, tenía esas piernas gordas y la cara también, y no me daban ganas de comer, no tenía aliento de nada, solo quería vivir acostada porque ni sentada ya casi no podía" $P(2), E(2), C E R$.

"Yo, todo lo que me conseguía, lo gastaba en médicos, yerbateros, brujos todo lo que me decían que me pudiera mejorar, yo iba" $(P)$ 4, (E) 4 HAP.
El tema común Pérdidas, describe los aspectos que marcan la vida de los enfermos debido a las implicaciones que trae la enfermedad renal y la diálisis peritoneal como su único tratamiento; las unidades de significado pérdida del rol, pérdida de la pareja y pérdida de la independencia, reflejan como la persona enferma se enfrenta a cambios drásticos que generaron frustración e incertidumbre, pero que a su vez les permitió crecer y fortalecerse como seres humanos. Se evidencia la lucha constante que se tienen con sigo mismos para aceptar su nueva condición, al no poder dar continuidad a lo que antes representaba su cotidianidad, ocasionando dolor al ver perdido 0 alterado su rol, así como las implicaciones de la dependencia económica o del requerimiento de un cuidador familiar para su terapia dialítica en algunos casos. Igualmente se vivencia la perdida de la pareja por miedo al rechazo o por abandono de la misma.

"Antes de mi enfermedad yo viajaba mucho, mi hijo es conductor de mula y él me convidaba a viajar con él y yo me iba, eso lo extraño mucho, ya que con la diálisis no puedo hacerlo" (P) 13, (E) 13, YMS.

"Yo trabajaba a diario yo hacía mis cosas en la casa y me iba a coger café y se llegó el día en que yo no me pude levantar de la cama" (P) 6, (E) 6, MA.

El tema común Corporalidad, representa la lucha que tiene el paciente frente a las manifestaciones de su enfermedad, los sentimientos ambiguos que se generan por los cambios en su aspecto físico y cómo logran auto reconocerse. Las unidades de significado Impacto del catéter, cambios en la apariencia física y reconociéndome otra vez, describen aspectos de temor y duda por el procedimiento quirúrgico de implantación del catéter y cómo su imagen física se ve afectada, al realizar cambios en la manera de vestir y cómo llevar el catéter sin que sea visible pero que no se ponga en riesgo su salud o la funcionalidad del dispositivo. Este proceso fue tortuoso, afectando su autoestima al sentirse menos bellas. Con el tiempo, la persona enferma logra asimilar los cambios físicos que se presentan con la enfermedad, aceptando el catéter peritoneal como parte de su cuerpo y su nueva imagen.

"Cuando me pusieron ese aparato ahí, yo me sentí mal, porque yo me sentía que no servía para nada, que no podía hacer nada, no me gustaba tocarme nada de eso" (P)5, (E)5 LEOO.

"Cuando a mí me pusieron el catéter por primera vez, yo me deprimí mucho, no me podía ver al espejo, me sentía muy fea" $(P) 16,(E) 16$, OJV. 
El tema común Limitaciones, se vivencia las falencias que tiene el servicio de salud del país, y las implicaciones que estas tienen frente a los usuarios que requieren de la terapia de remplazo renal de manera indefinida, las implicaciones que tiene para la persona enferma no tener continuidad en su atención y como ésta impacta negativamente en su automanejo. Las unidades de significado acceso al sistema de salud, cobertura y continuidad en la atención, demuestran la lucha que la persona enferma y su familia deben tener con las diferentes empresas prestadoras de salud, al sentirse vulnerados sus derechos de atención a nivel nacional, el no tener instituciones de mayor complejidad en las zonas rurales y profesionales debidamente capacitados para su manejo, así como la no continuidad en la atención de las unidades renales ya que representan un retroceso en el proceso de manejo de la diálisis, aumentando el riesgo de deserción del paciente 0 de complicaciones asociadas a la terapia.

"Yo sufría del azúcar pero como era difícil ir al médico yo solo iba a que me entregaran la insulina y ya, me la ponía en la casa pero nunca nos dijeron que me podía quedar ciega o que los riñones se dañaban" (P) 10, (E) 10 NJM.

"Cuando me cambiaron de unidad, me informaron que debían cambiarme el sistema de la diálisis y que tenía que volver aprender un nuevo procedimiento, eso me dio muy duro porque yo ya estaba acostumbrada, pero pues a uno le toca acostumbrarse" $(P) 14,(E)$ 14, YOT.

El tema común Soporte, representa la influencia de las fuentes generadoras de apoyo, su efecto amortiguador, frente a los eventos estresantes propios de la enfermedad y como estos favorecen al individuo y su calidad de vida. Las unidades de significado ayuda espiritual, encontrando una motivación para seguir y apoyo en el equipo de enfermería, resaltan la influencia positiva que tiene la creencia espiritual y el apoyo familiar para el afrontamiento de la enfermedad y la aceptación de la terapia de remplazo renal. Se evidencia la importancia de la intervención de Enfermería en el proceso de afrontamiento y seguimiento de la enfermedad, que incluye de forma satisfactoria en la adherencia del paciente a su tratamiento dialítico.

"Yo he, sido muy devota de Dios y pues él es que me ha dado la fuerza, para salir adelante, en cuanto a eso, le doy gracias a Dios por eso" (P)8, (E) 8 MJM.

"Y luego cuando empezaron a formarme acá, a explicarme la parte médica, la jefe lo que había ocurrido yo empecé a entender, empecé a tranquilizarme, porque me sentía en confianza" $(P) 13,(E) 13$ YMS.
El tema común Ganancias, describe la transformación que realiza el paciente luego de aceptar su condición de enfermedad y como logra involucrar su terapia dialítica a su vida, permitiéndole reincorporarse poco a poco a su cotidianidad. Las unidades de significado autonomía y cotidianidad, crecer espiritualmente, aprender el manejo de la diálisis, disfrutar- bienestar y uniendo la familia, describen como la persona enferma logra trascender ante la adversidad, el enfermo experimenta autonomía al tener una terapia ambulatoria permitiéndole realizar sus actividades diarias, se ve la importancia de fomentar el auto cuidado, la familia y el cuidado principal se convierten en un aliado para el profesional de Enfermería para que el sujeto de cuidado se re encuentre así mismo a través de la diálisis peritoneal.

"Yo ahora estoy normal, en la casa con mi marido y mis hijos allá en Pitalito, yo me conecto y me desconecto cuando yo quiera y así no tengo problemas, estoy pendiente de las cosas de la casa y de los animalitos que tenemos" (P) $11,(E) 11$ SS.

"Yo no me he muerto es por la diálisis, y ahí va uno, vengo a los controles me miran, y en la casa me hacen la diálisis todas las noches, yo me siento bien, como bien, duermo bien, estoy contento" $(P) 7,(E) 7 M C$.

Tabla 2. Caracterización socio demográfica de los participantes.

\begin{tabular}{|c|c|}
\hline Temas comunes & Unidades de Significado \\
\hline Enfrentando la Realidad & $\begin{array}{l}\text { Síntomas de la enfermedad } \\
\text { La diálisis como única opción. } \\
\text { Conductas de riesgo previas } \\
\text { La enfermedad como castigo }\end{array}$ \\
\hline Pérdidas & $\begin{array}{c}\text { Pérdida del rol } \\
\text { Pérdida de la pareja } \\
\text { Pérdida de la independencia }\end{array}$ \\
\hline Corporabilidad & $\begin{array}{c}\text { Impacto del catéter } \\
\text { Cambios en la apariencia física } \\
\text { Reconociéndome otra vez }\end{array}$ \\
\hline Limitaciones & $\begin{array}{l}\text { Acceso al sistema de salud } \\
\text { Cobertura y continuidad en la atención }\end{array}$ \\
\hline Soportes & $\begin{array}{l}\text { Ayuda espiritual } \\
\text { Encontrando una motivación para seguir } \\
\text { Apoyo en el equipo de enfermería }\end{array}$ \\
\hline Ganancias & $\begin{array}{l}\text { Autonomía y cotidianidad } \\
\text { Crecer Espiritualmente } \\
\text { Aprender el manejo de la diálisis } \\
\text { Disfrutar - Bienestar } \\
\text { Disfrutar - Bienestar } \\
\text { Uniendo la Familia }\end{array}$ \\
\hline
\end{tabular}

Fuente: Construcción de la autora. 


\section{Discusión y Conclusiones}

En la investigación se evidencia cómo es el proceso de afrontamiento, al inicio el enfermo busca posibles soluciones, para evitar o prorrogar el ingreso a diálisis, reconoce que su salud física está mal, al evidenciar los signos y síntomas que padece, pero no sabe que le está sucediendo, situación, que genera preocupación, dolor e impotencia, por lo cual buscan ayuda en las instituciones de salud, con el fin de encontrar una cura para sus dolencias; la necesidad de la diálisis, como única opción de tratamiento, es una noticia devastadora este proceso va acompañado de temores e incertidumbres por el riesgo de muerte y lo que podrá suceder en el futuro ${ }^{14,15}$.

Se buscan culpables por la condición, contemplándola incluso como un castigo divino, por causas del azar o del destino, hallazgos que coinciden con otros estudios con pacientes con enfermedades crónicas ${ }^{16,17}$.

Ahora bien, el participante ve la espiritualidad como un aliciente y le permite sobrellevar su enfermedad con resignación, Rodríguez et al, $2011^{18}$, señalan que la espiritualidad motiva y permite la búsqueda de trascendencia, propósito y sentido de vivir

Este padecimiento de enfermedad crónica, lleva al enfermo a enfrentar pérdidas de toda índole, en especial la de su rol, a nivel individual, familiar y social, generando sentimientos de tristeza y minusvalía, al sentirse no productivos, limitados para realizar sus actividades laborales, de estudio o desarrollar su rol en el hogar, como lo afirma Ledesma et al, $2007^{19}$.

Los participantes expresan cómo el proceso de enfermedad, afecta de manera negativa las relaciones socio afectivas, como la pérdida de la pareja, bien sea por que son abandonados, o porque son ellos, los que prefieren alejarse por temor a ser rechazados o juzgados por los cambios que sufren a nivel físico y psicológico y aquellos ajustes, que deben realizar por la terapia dialítica, esto se puede relacionar con lo expuesto por Morales et al, $2009^{20}$, los aspectos sociales son sustancialmente afectados por el tratamiento, incluyen cambios en el nivel del funcionamiento social y alteraciones en las relaciones matrimoniales y familiares.

Los miedos y la incertidumbre por la enfermedad y la terapia dialítica son permanentes, tal como lo señalan Cassareto et $\mathrm{a}^{21}$ y Araujo et $\mathrm{al}^{22}$. Los sentimientos, están relacionados con el impacto de tener el catéter peritoneal y cómo este cambia la apariencia física, genera dolor en el momento inmediato del procedimiento quirúrgico, y produce miedo por no saber cómo manejarlo y hacerlo parte de su cuerpo.

Para las mujeres el impacto en su estima y concepto es alto, precisan que deben cambiar su modo de vestir y acostumbrarse a su cuerpo, tal como lo describe Morales et $\mathrm{al}^{20}$.

En este proceso es vital el acompañamiento de la familia y del equipo interdisciplinario de la unidad renal, encabezado por el profesional de enfermería, quien sigue de la mano con el paciente, todo el proceso de enfermedad hasta que egresa de la institución médica para continuar su manejo en casa, tal como lo expone en Ruiz et al ${ }^{23}$.

Martínez et a ${ }^{24}$ reportan hallazgos similares en cuanto a las limitantes de acceso al sistema de salud, la no cobertura nacional y las demoras para acceder a las consultas con especialistas, exámenes especializados y tratamiento farmacológico, factor vital para garantizar la estabilidad del paciente y lograr evitar complicaciones.

La necesidad de un cuidador familiar, es inminente cuando el enfermo presenta algún tipo de limitación física o sensorial, lo cual to hace dependiente de alguien, quien ejecute el tratamiento de diálisis peritoneal en su hogar. En estos casos, es la familia quien se hacen cargo del cuidado, lo cual implica para ellos reorganizarse, establecer los posibles cambios en la dinámica familiar que les permita brindar y suplir las necesidades de su familiar, esto coincide con los resultados encontrados en la investigación de Reyes et al ${ }^{25}$.

Se resalta el impacto positivo, que tiene el soporte recibido por el equipo de salud, encabezado por enfermería, quien se encarga de la orientación de la terapia, educación frente al cuidado en casa, el entrenamiento del paciente y su familia, según la modalidad de diálisis al punto de consolidar un vínculo especial que busca facilitar la adherencia al tratamiento, fortalecer la motivación y el compromiso, empoderar al paciente en cuanto a la técnica de diálisis y el automanejo en el hogar, con miras a garantizar el éxito de la terapia, la calidad del vida del paciente y disminuir los factores de riesgo relacionados con el tratamiento dialítico, en concordancia con lo descrito por Periz et $\mathrm{al}^{26}$.

El participante logra aceptar la terapia de diálisis peritoneal como una opción de vida, que le permite reencontrarse poco a poco con su cotidianidad, seguir compartiendo con su familia e ir reconstruyendo su nuevo rol al 
vincularse de nuevo a su ambiente laboral o de estudios, permitiéndole mejorar su calidad de vida. Esto discrepa con los resultados obtenidos en el estudio de Araujo et al22, donde se describe que la vida del paciente se torna más pobre, y los días resultan más cortos, pues la mayor parte de ellos es dedicada a la terapia renal.

La principal limitación del estudio fue el acceso a los participantes, ya que estos se encontraban en su mayoría en zonas rurales lejanas de la unidad renal, sin embargo se lograron sortear para la recolección de la información.

A modo de conclusión podemos decir que la experiencia del paciente en diálisis peritoneal plantea la lucha interna que tiene el individuo, al enfrentar una enfermedad crónica. Para la persona enferma, cada día representa una victoria, una ganancia de poder compartir y seguir realizando lo que más les gusta, encontrar opciones de vivir lejos de las clínicas y ser autónomos en su tratamiento, les hace sentir útiles y productivos.

Es indispensable continuar realizando estudios cualitativos donde se puede indagar sobre la experiencia de los cuidadores familiares, quienes son el soporte para el individuo enfermo, así como lo es, la experiencia del profesional de enfermería quien asume un rol definitivo en todo el proceso de aceptación, entrenamiento, tratamiento y seguimiento del paciente.

\section{Financiación}

No se contó con ningún tipo de financiación económica para el desarrollo de la presente investigación.

\section{Agradecimientos}

Infinitas gracias a cada uno de los participantes por su vinculación en el proyecto, a la unidad renal de Clínica Medilaser Neiva y a la Universidad Nacional de Colombia.

Recibido: 16 mayo 2018

Revisado: 1 junio 2018

Modificado: 30 junio 2018

Aceptado: 3 julio 2018

\section{Bibliografía}

1. Barrera L, Carrillo GM, Chaparro L, Sanchez- B. Cuidado de Enfermería en situaciones de enfermedad crónica. Bogotá: Editorial Universidad Nacional de Colombia. 2014.

2. Lopera - Medina, M. La enfermedad renal crónica en Colombia: necesidades en salud y respuesta del Sistema General de Seguridad Social en Salud. Gerenc Polit salud Bogota. 2016;15:212-3.

3. Cuenta de alto costo. Situacion de la enfermedad renal cronica en colombia 2013. In: cundinamarca, editor. Santa fe de Bogota: Ministerio de salud y proteccion social;2013. p. 1-111.

4. Acosta - Hernández PA, Chaparro - López LC, Rey - Anacona C. Calidad de vida y estrategias de afrontamiento en pacientes con insuficiencia renal crónica sometidos a hemodiálisis, diálisis peritoneal o trasplante renal. Rev Colombiana de Psicologia. 2008;17:9-26.

5. Vergeles JM. En la enfermedad crónica, ¿la familia ayuda?, Revista AMF [Internet], 2013;9(9):495500.

6. Villarreal D, Angel J. Terapia Familiar sistemica: Una aproximacion a la teoria y la practica clinica. Revista de Avances en Psicología. 2015;1(1):4555.

7. Tejedor A, De las Cuevas Bou X. Cuidado paliativo en el paciente con Enfermedad renal crónico avanzado (grado 5) no suceptible a tratamiento dialítico, Rev Enf Nefrol. 2008;28,(3):129-36.

8. Ariza C. Soluciones de humanización en salud en la práctica diaria. Enferm. univ ENEO-UNAM. 2012; 9(1):41-51.

9. Ledón L. Enfermedades crónicas y vida cotidiana. Revista Cubana de Salud Pública. 2011;37(4):8899.

10. Solano MC. Fenomenología-hermenéutica y enfermería. Revista Cultura de los Cuidados. 2006; 10(19):5-6.

11. Organización Panamericana de la Salud y Consejo de Organizaciones Internacionales de las Ciencias 
Médica. Pautas éticas internacionales para la investigación relacionada con la salud con seres humanos, Cuarta Edición. Ginebra: Consejo de Organizaciones Internacionales de las Ciencias Médicas (CIOMS); 2016.

12. Noreña AL, Alcaraz $N$, Rojas GJ y Rebolldo D. Aplicabilidad de los criterios de rigor y éticos en la investigación cualitativa. Aquichan. 2012;12(3), 263-74.

13. Sánchez B. Fenomenología: un método de indagación para el cuidado de enfermería. En: Cuidado y práctica de enfermería. 2000. Bogotá: Universidad Nacional de Colombia.

14. Luque $E$, Barroso R, López M, Alapont M, Alconchel $S$, Torres $M$ et al. Diálisis peritoneal, la realidad subjetiva de convivir con una enfermedad crónica. Rev Soc Esp Enferm Nefrol. 2001;4(1),12-7.

15. Rivera A, Montero-López LM, Sandoval-Ávila R, Escala de Ansiedad ante la Muerte, de Templer: propiedades psicométricas en pacientes con insuficiencia renal crónica terminal. Journal of Behavior Health Social Issues. 2010; 2(2)83-91.

16. Rojas G. Estrategias de intervención psicológica en pacientes con cáncer de mama. Rev. Med. Clin. Condes. $2006 ; 17(4): 194-7$.

17. Rodríguez-Moctezuma JR, López-Delgado ME, Ortiz-Aguirre AR, Jiménez-Luna J, López-0caña LR, Chacón-Sánchez J. Etapas del Duelo en diabetes y control metabólico, Rev Med Inst Mex Seguro Soc. 2015;53(5):546-51.

18. Rodríguez $M$, Fernández $M L$, Pérez $M L$, Noriega R. Espiritualidad variable asociada a la resiliencia. Cuadernos hispanoamericanos de psicología.2011;11(2):24-49.
19. Rivera A, Montero M, Sandoval R. Desajuste psicológico, calidad de vida y afrontamiento en pacientes diabéticos con insuficiencia renal crónica en diálisis peritoneal. Salud mental. 2012;35(4):29-37.

20. Morales LM, Castillo E. Catheter meaning for the adolescents in dialysis, Rev. Colombia Médica. 2009; 40(3):316-22.

21. Cassaretto M, Paredes R. Afrontamiento a la enfermedad crónica: estudio en pacientes con insuficiencia renal crónica terminal. Revista de Psicología.2006;24(1):109-40.

22. Araujo-Sadala ML, Azevdo G, Regina E, Bucuvic E. La experiencia vivida por pacientes en diálisis peritoneal domiciliaria: un abordaje fenomenológico. Rev. Latino-Am. Enfermagem. 2012;20(1):1-8.

23. Ruiz R, Minguela J, Ocharán J, Gimeno I, Chena A. El entorno social de los pacientes en diálisis peritoneal. Nefrología. 2008;28(6),133-6.

24. León F, Ordoñez I, García D. Deficiencias en el tratamiento de pacientes diabéticos que terminaron en enfermedad renal crónica. Acta Médica Colombiana. 2007;32(2):57-67.

25. Reyes AG, Garrido A, Torres LE, Ortega P. Cambios en la cotidianidad familiar por enfermedades crónicas, Rev. Psicología y Salud. 2010;20(1):111-7.

26. Andreu L, Gruart P, Sánchez L. Visión enfermera de las necesidades psicosociales de los cuidadores de personas en tratamiento con Diálisis Peritoneal. Rev Soc Esp Enferm Nefrol. 2007;10(1):47-52.

Este artículo se distribuye bajo una Licencia Creative Commons Atribución-NoComercial 4.0 Internacional. https://creativecommons.org/licenses/by-nc/4.0/ 